\section{Congenital heart defects in Noonan syndrome and RIT1 mutation}

To the Editor: We read with great interest the article by Kouz et al. ${ }^{1}$ reporting on genotype-phenotype correlations of patients with Noonan syndrome (NS) heterozygous for RIT1 mutations. The authors performed an exhaustive analysis of the features associated with mutations in this gene, which was recently described as a new causative gene for the syndrome and accounts for approximately $5-10 \%$ of the total cases of NS cases. Cardiac and extracardiac features of this new subset of patients were discussed by Kouz et al., ${ }^{1}$ and clinical characteristics of patients with RIT1 mutation were compared to those found in patients with mutations in other genes known to be related to RASopathies.

We report here a few additional details regarding the cardiac phenotype in this subgroup of patients. Among our series of 161 patients with a molecularly confirmed diagnosis of RASopathy, 9 patients $(6 \%)$ with a clinical diagnosis of NS carrying a missense RIT1 mutation were recently identified. Congenital heart defects in this subgroup of patients are seen in $100 \%$ of patients, which is in line with the data reported by Kouz et al. ${ }^{1}$ Consistent with the published data, pulmonary valve stenosis, hypertrophic cardiomyopathy (HCM), atrial septal defect (ASD), and ventricular septal defect (VSD) were reported in our subjects with RIT1 mutations. One patient in our cohort had partial atrioventricular canal defect (pAVCD).

Four patients in our series presented HCM. Two of them had an obstructive HCM (oHCM), which was associated with subaortic stenosis and pulmonary valve stenosis in one patient who required an orthotopic heart transplant during the first year of life due to the severity of the cardiac phenotype, which was unresponsive to extensive medical treatment. These data confirm what was previously reported by Colan et al., ${ }^{2}$ as well as our unpublished data, documenting that patients with an early-onset HCM phenotype (younger than 2 years of age), particularly in cases of biventricular obstructions, have poor prognoses. Based on these considerations, HCM in NS subjects with a RIT1 gene mutation should be carefully evaluated, particularly if it occurs in early childhood.
Four patients had pulmonary valve stenosis with a typical thickened and dysplastic valve. The supravalvular region was involved in the lesion in all of them, as generally reported in NS. To the best of our knowledge, our patient with PAVCD is the first diagnosed with RIT1 mutation. This observation confirms the high prevalence of AVCD previously noted in RASopathies and not only in association with PTPN11 and RAF1 mutations. ${ }^{3}$

Finally, lymphatic anomalies were present in three out of the nine patients in our cohort, including chylothorax in two subjects and lymphedema of the lower limbs in one. Chylothorax was persistent in both children and required postoperative drainage. These data confirm the relevant role of RIT1 mutations in early-onset and late-onset lymphatic complications, which could lead to significant postoperative morbidity in these subjects.

In conclusion, the prevalence of congenital heart defects in patients carrying heterozygous RIT1 mutations is high and a great variety of anatomic types can be observed, including HCM, which can be particularly severe. Finally, complications due to lymphatic anomalies should also be taken into consideration.

\section{DISCLOSURE}

The authors declare no conflict of interest.

Giulio Calcagni, $M D, P h D^{1}$, Anwar Baban, $M D, P h D^{1}$, Francesca Romana Lepri, $P h D^{2}$, Bruno Marino, $M D^{3}$, Marco Tartaglia, $\mathrm{PhD}^{2}$ and Maria Cristina Digilio, $\mathrm{MD}^{2}$

${ }^{1}$ Department of Pediatric Cardiology and Cardiac Surgery, Bambino Gesù Children's Hospital, IRCCS, Rome, Italy; ${ }^{2}$ Genetics and Rare Diseases Research Division, Bambino Gesù Children's Hospital, IRCCS, Rome, Italy; ${ }^{3}$ Pediatric Cardiology, Department of Pediatrics, Sapienza University, Rome, Italy. Correspondence: Giulio Calcagni (giulio. calcagni@opbg.net)

\section{REFERENCES}

1. Kouz K, Lissewski C, Spranger S, et al. Genotype and phenotype in patients with Noonan syndrome and a RIT1 mutation. Genet Med e-pub ahead of print 21 April, 2016

2. Colan SD, Lipshultz SE, Lowe AM, et al. Epidemiology and cause-specific outcome of hypertrophic cardiomyopathy in children: findings from the Pediatric Cardiomyopathy Registry. Circulation 2007;115:773-781.

3. Digilio MC, Romana Lepri F, Dentici ML, et al. Atrioventricular canal defect in patients with RASopathies. Eur J Hum Genet 2013;21:200-204.

Advance online publication 29 September 2016. doi:10.1038/gim.2016.137 